\title{
Impact hydrologique d'un changement climatique sur le bassin du Rhône
}

\section{Impact of climate change on the hydrology of the Rhône catchment}

\section{Frédéric Hendrickx}

\author{
EDF R\&D - LNHE - 6, quai Watier 78401 CHATOU Cedex, France. \\ E-mail : trederic.hendrickx@edf.fr
}

Résumé. - Un modèle hydrologique a été déployé sur l'ensemble du bassin du Rhône permettant de mettre en relation la météorologie de l'ensemble du bassin versant français avec la ressource en eau.

L'utilisation conjointe de ce modèle hydrologique et de trois scénarios de changement climatique a permis de faire une première évaluation des tendances à attendre sur la disponibilité de la ressource en eau d'ici à 50 ans environ. Les trois scénarios de changement de climat utilisés pour ce travail indiquent tous une augmentation des températures moyennes sur l'année entre +2 et +3 "C. Cette élévation se décline sur l'année par une élévation en moyennes mensuelles interannuelles toujours supérieures $\dot{a}+1^{\circ} \mathrm{C}$ et pouvant atteindre ponctuellement $+5^{\circ} \mathrm{C}$ en fin d'été, début d'automne. Les simulations hydrologiques avec les trois scénarios climatiques utilisés convergent sur une baisse de près de $-50 \%$ des débits moyens mensuels en fin de période estivale. Sur les débits hivernaux, selon le scénario, cela se traduit par des évolutions pouvant aller de "aucune " modification à $+50 \%$ (en février) des débits moyens mensuels. Les simulations indiquent aussi une translation des régimes à influences nivales (forts débits en été) vers des régimes à influences pluviales (forts débits en hiver), conséquence de la modification du fonctionnement du manteau neigeux (moins de neige à moyenne altitude et moins longtemps). La lame d'eau annuelle devrait être légèrement diminuée.

\section{Mots-clés. - hydrologie, changement climatique, Rhône, France}

Abstract. - An hydrological model has been used on the whole of the Rhone basin linking the meteorology of the totality of French catchment with the water resource.

The joint use of this hydrological model with three climate change scenarios enabled a first assessment of forthcoming trends on water resource availability by the 2050 s. The three climate change scenarios used in this study all show an increase in the mean annual temperature from +2 to $+3^{\circ} \mathrm{C}$. This increase is expressed within the year by an interannual mean monthly rise always greater than +1 " $\mathrm{C}$, reaching up to $+5^{\circ} \mathrm{C}$ at the end of the summer, beginning of the autumn. Hydrological simulations using the three climate change scenarios all show a decrease of about $-50 \%$ of mean monthly dis- 
charge during the end of the summer. In winter, depending on the scenario, this corresponds to changes ranging from "nil" up to $+50 \%$ (in February) of mean monthly discharges. The simulations also show a change from snow-affected type flow regimes (large summer discharges) towards rain-fed type regimes (large discharges during winter), a direct consequence of the change in the snow cover (less snow at mean elevation, and during shorter periods). The runoff should decrease by a small amount.

Key words. - Hydrology, climate change, Rhone, France

\section{INTRODUCTION}

La communauté scientifique climatologue s'accorde à présenter le changement climatique comme tout à fait probable (IPCC, 2001). Elle annonce, de manière quasi unanime, que ce changement devrait être de grande ampleur, mais dans le même temps, elle ne sait pas trouver un accord sur la répartition spatiale et temporelle de ce changement à la surface du globe. Quoiqu'il en soit, des unités de recherche commencent à proposer des scénarios d'évolution du climat suite à une augmentation de la teneur en gaz à effet de serre dans l'atmosphère.

La disponibilité en eau revêt, pour les sites thermiques et nucléaires de production d'électricité, un caractère essentiel quant à la disponibilité de la source froide, mais aussi pour les barrages hydroélectriques quant aux capacités de production. EDF R\&D a donc engagé dès 1994 des travaux qui avaient pour objectif de proposer des scénarios possibles d'évolution de la contrainte hydrique dans le cas d'un réchauffement de l'atmosphère en essayant de traiter le probleme à la fois sous l'angle du volume (débit) et sous l'angle thermique (échauffement des eaux).

Afin de tenir cet objectif, nous nous sommes appuyés sur des partenariats avec deux équipes de climatologues français à savoir celle du CNRS - Laboratoire de Météorologie Dynamique (LMD) et celle de Météo France - Centre National de Recherche Météorologique (CNRM). Ces partenariats ont permis la constitution et la récupération de trois scénarios de changement climatique permettant d'envisager un travail sur l'évolution de la contrainte hydrique dans le cadre d'un réchauffement climatique.

Un travail de modélisation, via un modèle hydrologique de l'ensemble du bassin versant français du Rhône, a permis d'envisager l'évolution moyenne de la disponibilité en eau en quelques points du bassin versant du Rhône. Ce travail constitue une première étape dans l'analyse des évolutions possibles de la source froide.

Cet article est consacré à l'approche retenue et aux résultats obtenus pour appréhender les évolutions moyennes de la disponibilité en eau, sous l'hypothese d'un doublement de $\mathrm{CO}$, dans l'atmosphère, ce qui devrait 
correspondre à un horizon d'environ 50 ans en tenant compte de l'ensemble des émissions de gaz à effet de serre.

\section{SCENARIOS CLIMATIQUES}

\subsection{Hypothèses}

Au-delà de la limite de prédictibilité, toute prévision déterministe est exclue mais il reste l'espoir de prévoir la caractéristique moyenne du temps, c'est-à-dire le climat.

Peut-on prévoir le climat un mois, une saison, une année à l'avance? Peut-on espérer prévoir l'effet, sur le climat du siècle prochain, de l'enrichissement de l'atmosphère en gaz à effet de serre? Les modèles de circulation générale essayent d'apporter des réponses à ces diverses questions.

La prise en compte dans ces modèles du contenu chimique et des propriétés radiatives du gaz atmosphérique, les rend aptes à représenter l'impact de l'effet de serre d'origine anthropique sur le climat. Pour disposer d'éléments de comparaison entre les différentes simulations du climat, il est nécessaire de formuler une hypothèse sur l'évolution du contenu de l'atmosphère en gaz à effet de serre. La difficulté actuelle, dans les modèles de circulation générale, de prendre en compte l'ensemble des gaz à effet de serre dans l'atmosphère, a conduit à formuler une hypothèse en équivalent $\mathrm{CO}_{2}$ correspondant à un doublement de la concentration en $\mathrm{CO}$, dans l'atmosphère. $\mathrm{Ce}$ doublement en équivalent $\mathrm{CO}_{2}$, avec les prospectives actuelles sur les émissions de gaz à effet de serre, devrait correspondre à un horizon de 50 ans.

En fait, le doublement réel de la concentration du seul $\mathrm{CO}_{2}$ n'aurait lieu que dans 200 ans (hypothèse de croissance exponentielle à partir des chiffres de S. Joussaume, 1993: $353 \mathrm{ppm}$ en 1990, $400 \mathrm{ppm}$ dans 25 ans et 500 ppm dans 100 ans).

\subsection{Présentation des scénarios}

\subsubsection{Scénarios “ LMD1 》 et “ LMD2 "}

Deux scénarios ont été constitués, par EDF, à l'aide du modèle de circulation générale du CNRS - Laboratoire de Météorologie Dynamique. Ces deux scénarios se distinguent par des raffinements différents du maillage du modèle. Le premier divise les cercles de latitude en 64 mailles, les demi-cercles de longitude en 50 mailles et dispose de 11 points de grille en altitude. Le second scénario est une version plus raffinée, avec 96 mailles sur les cercles de latitude, 72 sur les demi-cercles de longitude et 15 points de grille en altitude.

Les simulations du changement climatique sous un doublement du $\mathrm{CO}$, ont été contraintes aux limites par les températures de la mer issues du modèle couplé du Max Planck Institute de Hambourg. 
Les simulations ont été réalisées par EDF dans le cadre du programme européen Environnement (Parey, 1996). Ainsi, nous disposons de deux scénarios de changement climatique nous indiquant les anomalies climatiques (i.e. les écarts à la moyenne mensuelle interannuelle sur la température et les précipitations entre la période de simulation et la période de référence) attendues sous un doublement de $\mathrm{CO}_{2}$ dans l'atmosphère. Ces deux scénarios seront appelés par la suite LMD1 pour le moins raffiné et LMD2 pour le plus raffiné des deux.

\subsubsection{Scénario “ ARPEGE »}

Météo France (Centre National de Recherche Météorologique) a développé le modèle ARPEGE Climat.

La particularité du modèle ARPEGE Climat est de pouvoir travailler sur une grille irrégulière. $\mathrm{Ce}$ modèle a la particularité d'être un modèle spectral, c'est-à-dire que la discrétisation horizontale des équations de Navier-Stokes est effectuée par projection sur la base des harmoniques sphériques. Le lien avec la position géographique se fait en utilisant une grille de Gauss de résolution $192 \times 96$ (il s'agit de latitudes au sens de Gauss). On peut noter que les mailles sont très irrégulières : $60 \mathrm{~km}$ de côté sur la mer Tyrrhénienne et $700 \mathrm{~km}$ aux antipodes.

La création du scénario dit ARPEGE a été faite en utilisant les températures de surface de la mer du modèle couplé du Hadley Center.

La France métropolitaine est représentée par 135 points environ. A titre de comparaison, dans une maille du scénario LMD2, on peut placer environ une vingtaine de mailles du scénario ARPEGE. Ce modèle est donc plus détaillé, sur la France, que les modèles LMD.

\subsection{Premier résultat des trois simulations : le réchauffement climatique}

Les trois simulations du climat diffèrent à la fois par le modèle utilisé, le forçage océanique, le raffinement du maillage. Ces différences se traduisent par un réchauffement plus important dans les calculs avec le modèle ARPEGE Climat que dans les calculs avec le modèle du LMD.

Le réchauffement obtenu, suite à un doublement de la concentration du $\mathrm{CO}_{2}$ atmosphérique, donnerait une élévation en moyenne sur l'année de la température de +2 à $+3^{\circ} \mathrm{C}$ sur l'ensemble du bassin versant français du Rhône (fig. 1).

Cette évolution se déclinerait sur l'année par une élévation en moyenne mensuelle interannuelle toujours supérieure à $+1{ }^{\prime \prime} \mathrm{C}$ et pouvant atteindre ponctuellement $+5{ }^{\circ} \mathrm{C}$ en fin d'été, début d'automne dans le cas du scénario ARPEGE. 


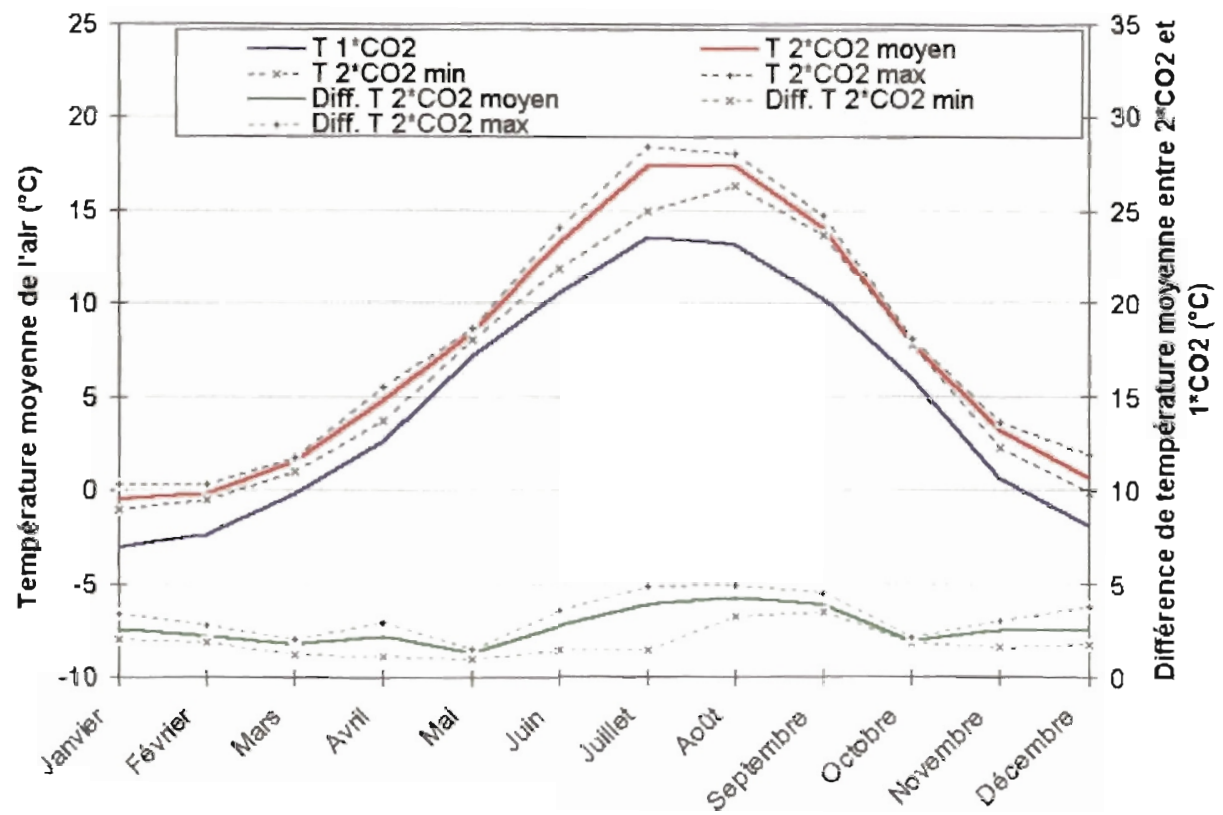

Fig. 1. - Évolution de la température de l'air sur le sous bassin de Saint Gervais le Port (en rouge: moyenne des trois scénarios - en pointillé noir: minimums et les maximums sur les trois scénarios).

Fig. 1. - Change in air temperature for the catchment of St Gervais le Port (red: average of the three scenarios - dashed lines : minimum and maximum of the three scenarios).

\section{MODÉLISATION HYDROLOGIQUE}

\subsection{Bassin du Rhône}

Le bassin du Rhône représente environ $71000 \mathrm{~km}^{2}$ à Viviers. II se caractérise par des situations géographiques et climatiques très variées. Du point de vue morphologique, on peut distinguer les plaines (Saône supérieure, sortie du couloir Rhodanien à l'embouchure), les montagnes issues de l'orogenèse hercynienne, longtemps érodées (Massif Central, Vosges) et les montagnes jeunes issues de l'ère tertiaire (Alpes, Jura). Du point de vue géologique, le sol est également assez diversifié, même si les séries sédimentaires dominent (non plissées dans les plaines, plissées dans les montagnes jeunes). Du point de vue climatologique, on retrouve les climats de montagne dans le Massif Central et les Alpes, les influences continentales dans le nord, les influences océaniques au nord-ouest et le climat méditerranéen au sud. 


\subsection{Modèle hydrologique}

\subsubsection{Le choix du modèle}

Le bassin du Rhône entraîne, de par sa dimension et ses caractéristiques, plusieurs contraintes dans le cadre d'une modélisation hydrologique :

- la superficie du bassin nous oblige à choisir un modèle pragmatique. Les aspects pré- et post-traitements sont primordiaux pour la mise en œuvre de l'outil sur un aussi grand domaine (utilisation des SIG, des MNT, analyse de base de données),

- la forte diversité du relief, du climat, de la géologie, et donc du comportement hydrologique, ont imposé le choix d'un modèle spatialisé (en $X, Y$ mais aussi en $Z$ pour tenir compte de l'influence de l'altitude sur les régimes de température et de précipitation),

- la présence de stocks de neige importants durant l'hiver (dans les Alpes notamment) est à prendre en compte dans la modélisation. Ces stocks sont d'autant plus importants qu'ils seront sans doute à l'origine de modifications conséquentes de l'hydrologie suite à un réchauffement climatique.

Le modèle retenu a été finalement le modèle issu de I'INRS-Eau (Québec) nommé CEQUEAU (Charbonneau et al., 1977). Ce modèle est un modèle conceptuel qui permet de simuler la quantité et la qualité de l'eau en rivière. Dans le cadre de ce travail, seul le volet simulation de la quantité a été abordé.

\subsubsection{Le Modèle CEQUEAU}

CEQUEAU est un modèle à bilans qui permet de prendre en compte certaines caractéristiques physiques d'un bassin versant et leurs variations dans l'espace et dans le temps. Ce modèle s'appuie sur une discrétisation de l'espace en éléments carrés, eux-mêmes subdivisés par les lignes de partage des eaux (fig. 2). Le modèle permet ainsi de simuler les débits aux points de jaugeage ainsi qu'en tout autre point du bassin versant. Le pas de temps utilisé est le jour.

Les entrées du modèle sont :

- la géographie du bassin versant (Iimite du BV, réseau de drainage, MNT),

- les précipitations solides et liquides journalières, les températures minimales et maximales journalières. Ces variables nous sont données par les stations pluviométriques et thermométriques associées au bassin,

- les fichiers de paramétrisation de la modélisation (pas de temps de calcul, paramètres du modèle CEQUEAU). Dans ces derniers, on pourra distinguer des paramètres physiques (calculables) et des paramètres calés (par essai-erreur).

Le modèle permet d'obtenir les valeurs de calcul de débit aux stations débimétriques de référence mais aussi en d'autres points du bassin. II permet aussi d'avoir accès à des 

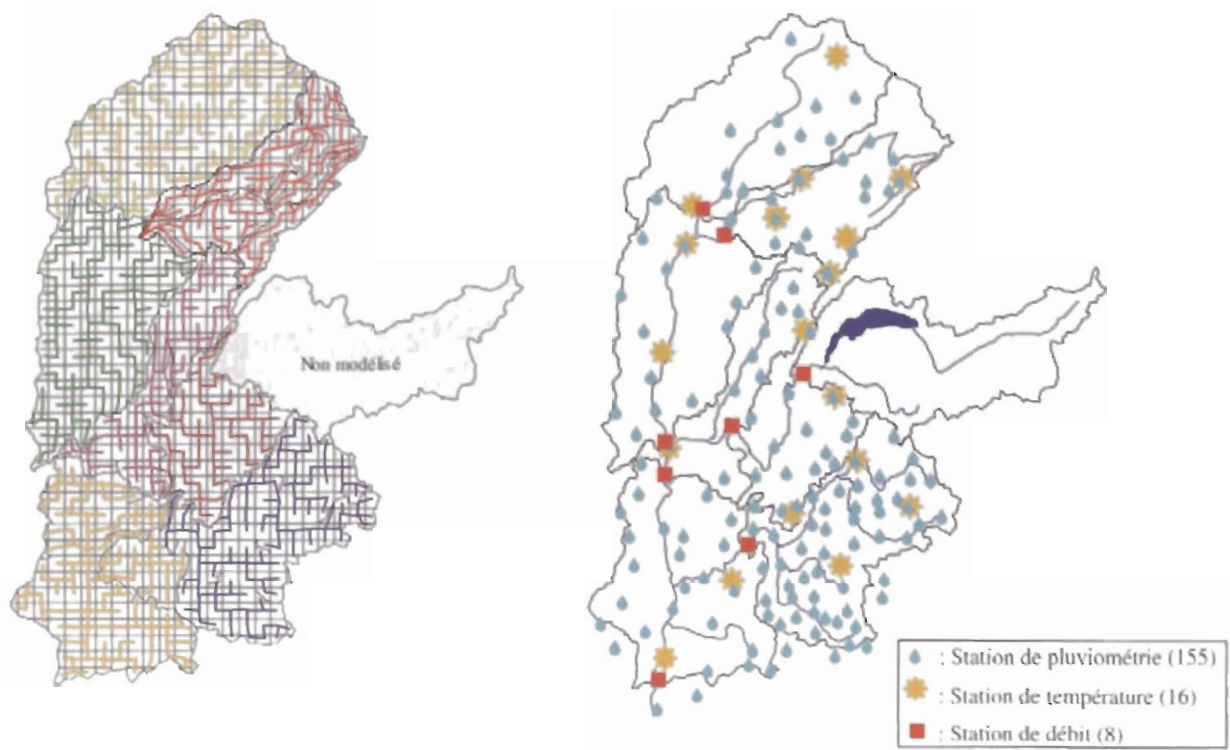

Fig. 2. - Maillage ei Base de données du módéle Cequeau sur le Rhône.

Fig. 2. - Grid mesh and data base of Cequeau model over the Rhone catchment.

" cartographies" de l'état des variables d'entrée et des variables internes du modèle (pluie, neige, évapotranspiration (ETP), stock de neige, stock d'eau du bassin versant).

Le modèle tient compte d'une spatialisation des variables météorologiques à l'aide de multiples stations météorologiques sur un même bas$\sin$. Les valeurs des variables météorologiques sont ajustées en fonction de la différence d'altitude entre la maille et la station associée. Ces modifications se font à l'aide de gradients de température $(T=f(z))$ et de pluviométrie $(P=g(z))$ selon l'altitude. Ces facteurs sont calculés à partir des stations de référence disponibles sur le bassin.
Le calcul se fait en deux étapes. La première concerne le calcul de la production de chaque maille, à savoir l'eau qui sera disponible pour l'écoulement, et la seconde porte sur le transfert de cette eau au sein du bassin versant.

La production est modélisée par un système de réservoirs (fig. 3). Ces réservoirs se traduisent de manière paramétrique par des coefficients de vidange et des seuils de vidange. Ce sont ces paramètres essentiels qu'il convient d'ajuster lors du calage du modèle. Ce sont eux qui gèrent l'équilibre dynamique entre ETP, stock de neige, ruissellement et infiltration. Chacun de ces paramètres est uniforme sur tout le sous bassin modélisé. 


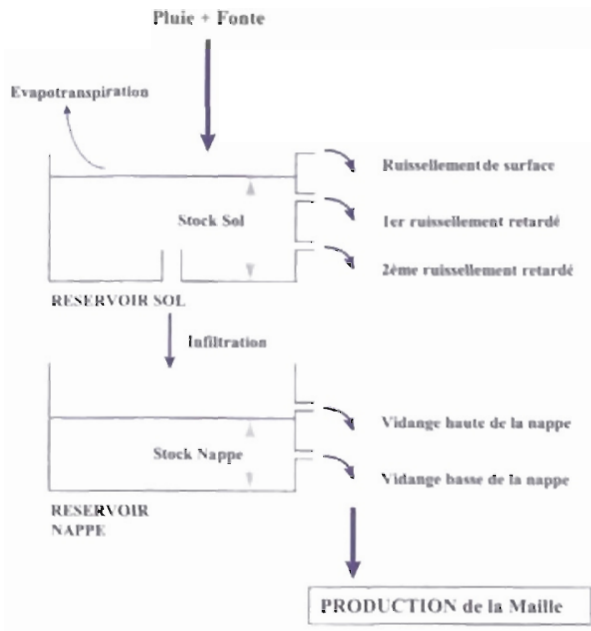

Fig. 3. - Schéma de production d'une maille CEQUEAU.

Fig. 3. - Sketch of the production module of one grid in CEQUEAU.
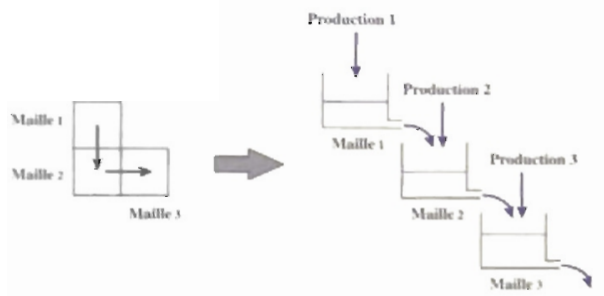

Fig. 4. - Schéma de transfert du modèle CEQUEAU.

Fig. 4. - Sketch of the routing module in CEQUEAU.

Le transfert des volumes produits sur chaque maille est ensuite assuré en tenant compte de la connectivité des mailles. La transformation, dans le temps et dans l'espace, d'un débit entre deux mailles est réalisée à l'aide d'un réservoir et d'un coefficient de vidange (fig. 4). Le transfert de ces volumes d'eau jusqu'à l'exu- toire se fait donc par une cascade de simples réservoirs caractérisés par un coefficient de vidange.

Le calage s'attache à restituer par ordre de priorité :

- la lame d'eau annuelle observée,

- les lames d'eau mensuelles observées,

- les minimums et maximums observés pour chaque épisode pluvio. métrique.

Les qualités d'un calage d'un modèle hydrologique résident essentiellement dans sa capacité à être à la fois bon sur les étiages et bon sur les fortes crues (fig. 5). Cette dernière qualité est souvent la plus difficile à obtenir et dépend fortement de la qualité du modèle lui-même.

Les critères traduisants la qualité du calage sont nombreux. Nous retenons aujourd'hui le plus classique: le critère de Nash. (Nash et al., 1970).

$$
\mathrm{NASH}=1-\frac{\sum_{1}\left(q_{c a l},-q_{o b s}\right)^{2}}{\sum\left(q_{o b s},-\bar{q}_{o b s}\right)^{2}}
$$

où $q_{c a l}$ est le débit calculé à l'exutoire le jour $i, q_{o b s}$ est le débit réel observé à l'exutoire le jour i et $\bar{q}_{o b s}$ est le débit moyen interannuel observé à l'exutoire.

Pendant la phase de calage, il apparaît souvent intéressant de pouvoir comparer l'hydrogramme calculé à celui réellement observé. Cette observation est riche sur la connaissance du comportement hydrologique du bassin versant. Elle apporte des indications sur les paramètres à 
corriger pour améliorer la qualité de la simulation.

La première année de simulation n'est jamais prise en compte dans la qualité du calage. C'est une année qui sert à l'initialisation des différents stocks du modèle.

La validation du modèle se fait sur une période différente de celle du calage. Elle se fixe pour objectif de tester la robustesse du calage et de vérifier que les années de référence du calage ne sont pas trop singulières.

On considère, usuellement, qu'un critère de Nash supérieur à 0,8 correspond à un calage de qualité et qu'une dégradation de ce critère inférieure ou égale à 0,1 lors de la validation de ce critère indique une bonne robustesse du modèle.

\subsection{Application au Rhône}

Le bassin versant français du Rhône a été découpé en sept sous bassins versants (fig. 6). Ce découpage s'appuie sur des stations cle débit existantes. II a eu pour objectif de s'approcher au mieux des quatre sites nucléaires présents sur l'axe Rhône (Bugey, Saint-Alban, Cruas, Tricastin), tout en essayant de respecter les particularités climatiques

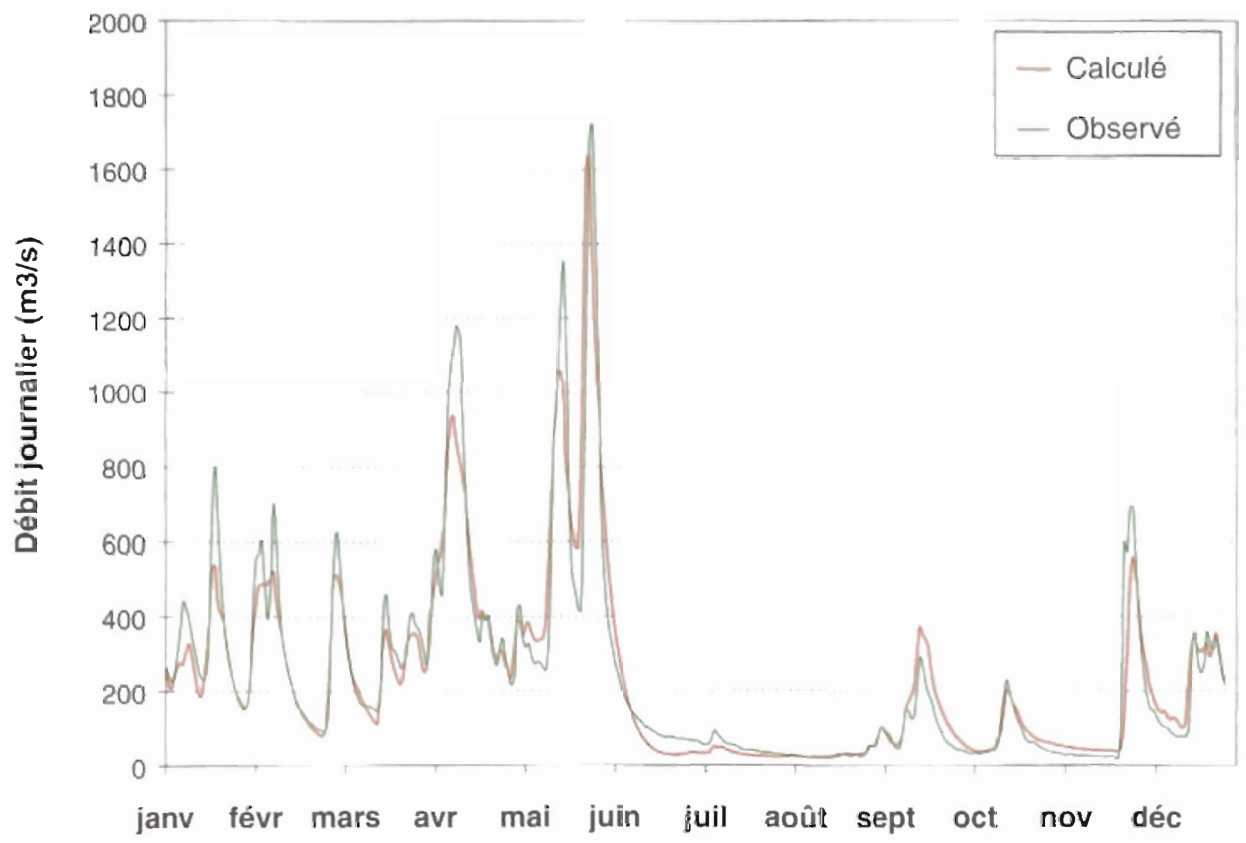

Fig. 5. - Exemple de calage (Station de Neublans (Doubs) - année 1983 - Indice de Nash de 0,9 sur 25 ans).

Fig. 5. - Example of fitting (station Neublans (Doubs) - year 1983 - Nash criterion of 0.9 over 25 years). 


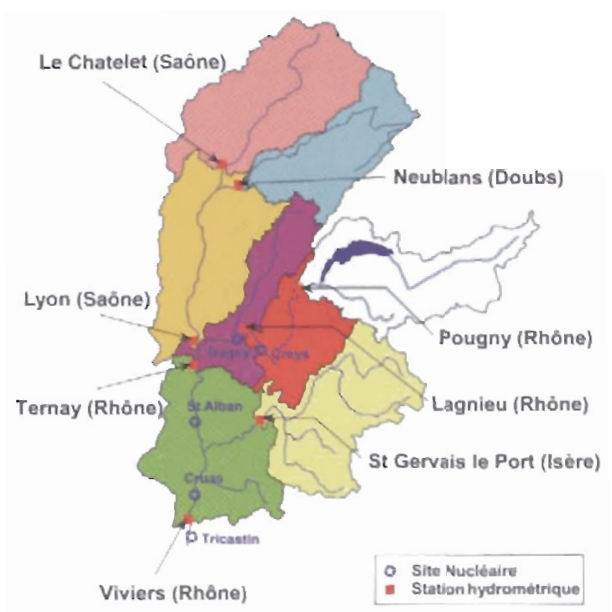

Fig. 6. - Découpage en 7 sous bassins versants du bassin français du Rhône.

Fig. 6. - The seven sub-catchments of the French part of the Rhone catchment.

et géographiques du bassin du Rhône.

Chaque sous bassin versant prend en apports, le calcul des sous bassins amonts.

Si l'on fait un zoom sur deux stations de référence, Saint Gervais le Port (exutoire d'un grand bassin Alpin) et Viviers (exutoire de l'en- semble du domaine étudié), on peut voir (tableau I), des résultats assez différents mais de bonne qualité.

La station de référence de Viviers est très bien reproduite par le modèle complet, avec un critère de Nash de 0,94 sur la période de calage et de 0,93 sur la période de validation. Cette faible dégradation du critère de Nash sur la période de validation permet d'espérer une très bonne robustesse du modèle. Par contre, la station de référence de Saint Gervais le Port est de qualité moins bonne avec un critère de Nash sur les valeurs journalières de 0,72 sur la période de calage et de 0,68 sur la période de validation. Ce résultat s'explique essentiellement par la présence de nombreux ouvrages hydroélectriques qui perturbent les régimes hydrologiques au pas de temps journalier mais aussi mensuel. On retrouve les éléments de cette perturbation dans l'écart maximum sur les débits mensuels interannuels (tableau I) qui atteint $13,1 \%$ et $13,9 \%$, sur respectivement la période de calage et la période de validation, tandis que la lame d'eau

Tableau I. - Synthèse partielle de la qualité et de la robustesse des modèles sur l'Isère et sur le Rhône à Viviers.

Table I. - Partial summary of the quality and robustness of the models over the Isere river and the Rhône at Viviers.

\begin{tabular}{|l|c|c|c|c|}
\hline & \multicolumn{2}{|c|}{ Saint Gervais le Port } & \multicolumn{2}{c|}{ Viviers } \\
\hline & Calage & Validation & Calage & Validation \\
\hline $\begin{array}{l}\text { Ecart lame d'eau annuelle } \\
\text { Calculée / Observée (\%) }\end{array}$ & $1.2 \%$ & $2.7 \%$ & $-3.0 \%$ & $9.0 \%$ \\
\hline Critère de Nash journalier & 0.72 & 0.68 & 0.94 & 0.93 \\
\hline Critère de Nash mensuel & 0.84 & 0.83 & - & - \\
\hline $\begin{array}{l}\text { Ecart maximum débit mensuel } \\
\text { interannuel (\%) }\end{array}$ & $13.1 \%$ & $13.9 \%$ & $2.4 \%$ & $6.8 \%$ \\
\hline
\end{tabular}


annuelle est très bien reproduite $(1,2 \%$ et $2,7 \%$ d'écart entre le calculé et l'observé). La prise en compte dans la phase de calage de l'observation du nombre de jours d'enneigement à différentes altitudes a permis de suppléer aux imperfections de la donnée débimétrique. On retrouve, malgré ces imperfections, un modèle qui présente très peu de dégradations entre la période de calage et la période de validation, ce qui là encore permet d'espérer une bonne robustesse du modèle.

La robustesse se trouve encore confirmée par la comparaison des régimes moyens sur les deux périodes (fig. 7 et fig. 8). On constate que les régimes moyens sur ces deux périodes sont assez différents, avec par exemple dans le cas de Viviers une forte dominante nivale sur la période de validation, tandis que la période de calage présentait plus une dominante pluviale.

Les courbes de débits classés sur les deux périodes de calage et de validation permettent de constater, premièrement, la capacité du modèle à bien reproduire la distribution fréquentielle de débit, à savoir bien reproduire les crues et les étiages dans leur ensemble, et en second lieu, la faible dégradation de cette restitution entre la période de calage et la période de validation.

\section{SIMULATIONS SOUS CHANGEMENT CLIMATIQUE}

\subsection{Constitution des forçages météorologiques}

Les trois scénarios climatiques ont des pas d'espace et un pas de temps qui leur sont propres. L'information climatique de ces scénarios est distribuée sur des mailles de plusieurs dizaines de kilomètres et au pas de temps mensuel. L'hydrologue travaille pour sa part avec des données plus détaillées (maille de quelques kilomètres et pas de temps journalier). Ces différences d'échelles spatiales et temporelles imposent, avant la simulation hydrologique des impacts, de préciser le scénario climatique par une désagrégation de l'information climatique. Le problème se pose, par exemple, quand il s'agit d'interpréter le résultat d'un scénario climatique qui donnerait une anomalie de $+10 \%$ de précipitation: est-ce $10 \%$ plus intense ou plus longtemps? Pour l'hydrologue, cette distinction est essentielle. Les climatologues s'accordent aujourd'hui à dire que l'évolution du climat se traduirait essentiellement par des variations d'intensité des précipitations, réparties sur un même nombre de jours (Martin, 1995). Les chroniques de précipitation et de température seront donc obtenues en appliquant des coefficients de corrections mensuelles aux chroniques actuelles (méthode dite " des perturbations" (Reynard et al., 2001) 

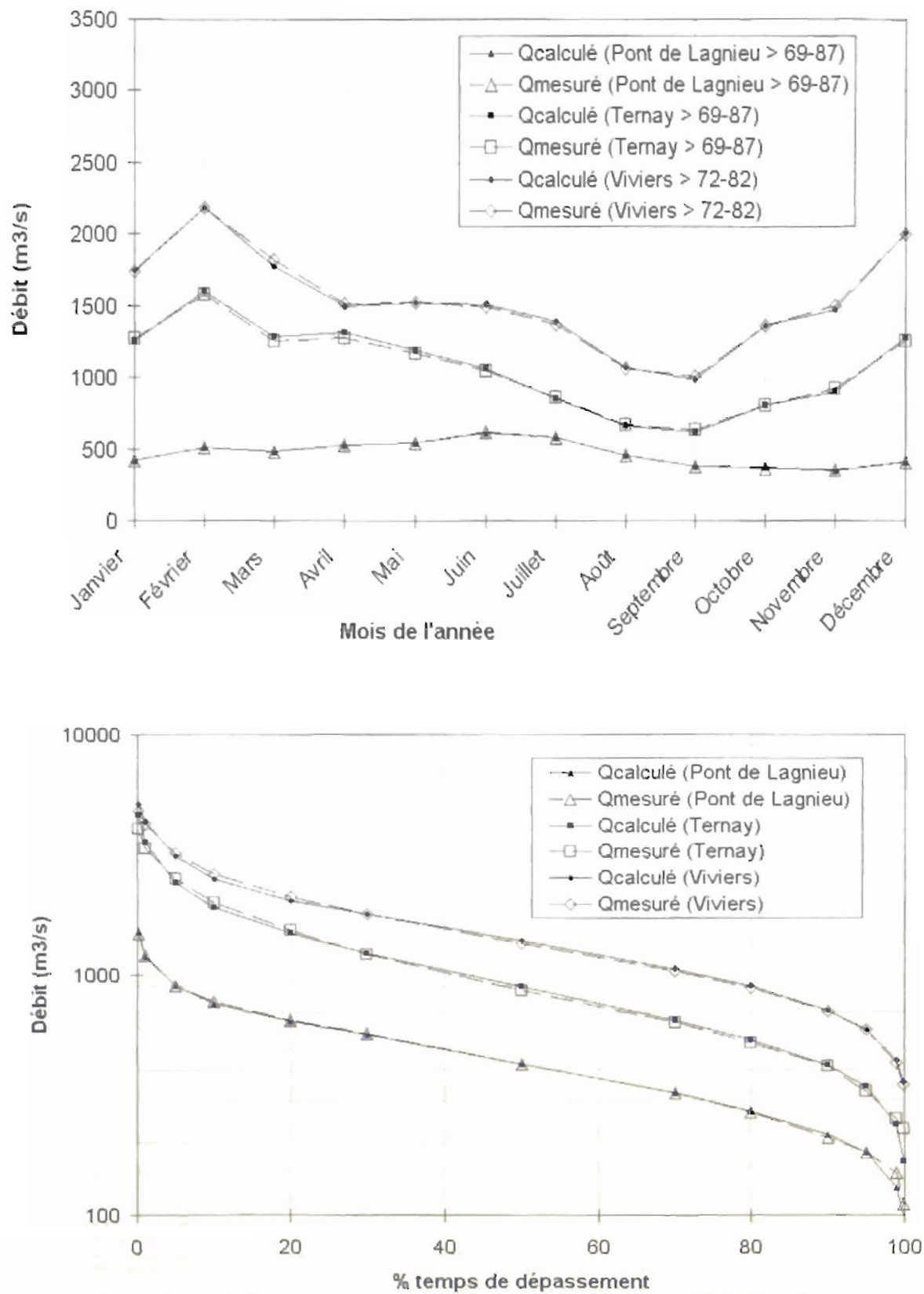

Fig. 7. - Comparaison a Calculé-Observé n sur la période de calage (débits classés en bas - débits mensuels interannuels en haut) en trois stations.

Fig. 7. - Comparison " simulated-observed" over the fitting period (bottom : Flow-duration-curves up : mean monthly flows). 
Impact hydrologique d'un changement climatique sur le bassin du Rhône
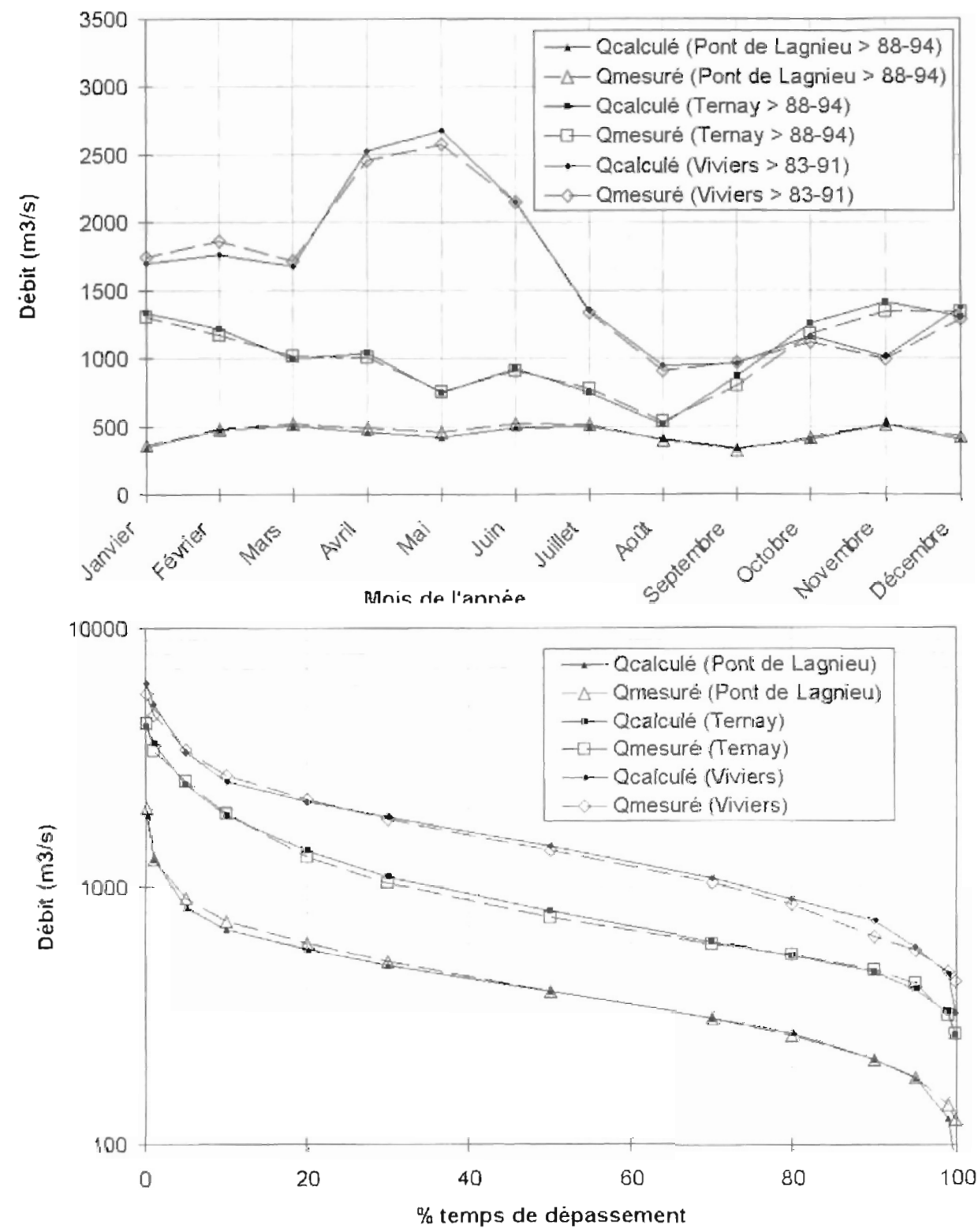

Fig. 8. - Comparaison "Calculé-Observé " sur la période de validation (débits classés en bas - débits mensuels interannuels en haut).

Fig. 8. - Comparison "simulated-observed" over the validation period (bottom : flow-duration-curves - up : mean monthly flows). 
Les simulations sous $2^{\star} \mathrm{CO}_{2}$ seront faites à partir des modèles hydrologiques calés sur chaque sous bassin versant et de nouvelles séries chronologiques de précipitation et de température. Ces nouvelles séries chronologiques seront obtenues à partir des résultats des 3 scénarios climatiques (LMD1, LMD2, ARPEGE) ce qui permettra d'avoir trois jeux de forçages météorologiques. La modification des débits sous $2{ }^{*} \mathrm{CO}_{2}$ sera analysée par comparaison avec les valeurs calculées par le modèle CEQUEAU sous climat observé (noté ici $1{ }^{*}\left(\mathrm{O}_{2}\right)$.

Pour chaque simulation d'un sous bassin versant sous $2^{*} \mathrm{CO}_{\nu}$, nous présenterons successivement l'impact sur la température moyenne de l'air du sous bassin versant, l'impact sur la lame d'eau annuelle écoulée à l'exutoire, l'évolution de la répartition interannuelle des débits et enfin une évolution possible des statistiques de débit (crue et période de retour, durée des étiages). Ces dernières analyses seront limitées par les données utilisées pour le calage et la validation sous $1{ }^{\star} \mathrm{CO}_{2}$ et pour la simulation sous $2^{\star} \mathrm{CO},(25$ années au total). Elles n'auront donc qu'un sens relatif, en ne permettant de voir que l'évolution en fréquence du risque crue et du risque étiage. Elles ne peuvent constituer, par la petite taille de l'échantilIon de données de débit, une évaluation fiable en absolu pour des fréquences rares.

\subsection{Résultats}

\subsubsection{Saint Gervais le Port : Évolution de l'hydrologie Alpine}

Les trois scénarios climatiques utilisés indiquent une augmentation moyenne de la température de l'air de 2,1 à $2,9^{\circ} \mathrm{C}$ sur le sous bassin versant de l'Isère (tableau II).

Cette augmentation de température se décline dans l'année par une élévation de température toujours supérieure à 1 " $\mathrm{C}$ et pouvant atteindre des valeurs de 4 à $5{ }^{\circ} \mathrm{C}$ d'élévation sur la période estivale.

Les lames d'eau annuelles écoulées seraient peu modifiées ou un peu réduites (tableau III). Le scénario ARPEGE n'entraîne, en effet, quasiment pas de variation de la lame d'eau annuelle $(-0.9 \%)$; par contre, les scénarios LMD engendreraient une diminution significative de l'ordre de $10 \%$ (8,7 à $12,2 \%)$.

La répartition sur l'année de la lame d'eau écoulée serait, par contre, beaucoup plus marquée. L'augmentation de la température impacterait fortement le régime nival en modifiant à la fois la nature des précipitations (plus sous forme liquide) et le fonctionnement du manteau neigeux (en moyenne -30 à -50 jours d'enneigement à $1500 \mathrm{~m}$ ). On constate (fig. 9) un avancement de 1 mois de la période des "fortes eaux", un étiage hivernal moins marqué et un étiage automnal plus sévère.

Les plus fortes augmentations de débit sont enregistrées pour les mois de février à juin avec une grande dis- 
Tableau II. - Températures et variations en ${ }^{\circ} \mathrm{C}$ mensuelles moyennes du sous bassin de l'Isère à Saint Gervais le Port.

Table II. - Temperature and changes in ${ }^{1} \mathrm{C}$ monthly means of the Isère sub-catchment at Saint Gervais le Port.

\begin{tabular}{|c|c|c|c|c|c|c|c|}
\cline { 2 - 8 } \multicolumn{1}{c|}{} & \multicolumn{4}{c|}{ Température movenne } & \multicolumn{3}{c|}{ Variation de température / 1CO2 } \\
\cline { 2 - 9 } \multicolumn{1}{c|}{} & $1 \mathrm{CO2}$ & ARPEGE & LMDI & LMD2 & ARPEGE & LMDI & LMD2 \\
\hline Janvier & -3.1 & -0.6 & 0.3 & -1.1 & 2.5 & 3.4 & 2.1 \\
\hline Février & -2.4 & -0.5 & 0.3 & -0.3 & 1.9 & 2.8 & 2.1 \\
\hline Mars & -0.2 & 1.8 & 1.0 & 1.6 & 2.0 & 1.2 & 1.8 \\
\hline Avril & 2.6 & 5.5 & 4.2 & 3.7 & 2.9 & 1.6 & 1.1 \\
\hline Mai & 7.1 & 8.7 & 8.1 & 8.3 & 1.5 & 0.9 & 1.2 \\
\hline Juin & 10.5 & 14.1 & 13.0 & 11.9 & 3.5 & 2.4 & 1.4 \\
\hline Juillet & 13.6 & 18.4 & 17.9 & 15.0 & 4.8 & 4.3 & 1.4 \\
\hline Août & 13.2 & 18.1 & 16.4 & 17.0 & 4.9 & 3.2 & 3.8 \\
\hline Septembre & 10.2 & 13.9 & 13.7 & 14.8 & 3.7 & 3.5 & 4.5 \\
\hline Octobre & 5.9 & 7.8 & 7.7 & 8.0 & 1.9 & 1.8 & 2.1 \\
\hline Novembre & 0.6 & 3.4 & 3.7 & 2.3 & 2.7 & 3.0 & 1.6 \\
\hline Décembre & -1.9 & 0.4 & 1.9 & -0.2 & 2.3 & 3.8 & 1.8 \\
\hline Année & 4.7 & 7.6 & 7.3 & 6.7 & 2.9 & 2.7 & 2.1 \\
\hline
\end{tabular}

Tableau III. - Évolution des débits mensuels interannuels à la station de Saint Gervais le Port. Table III. - Change in the mean monthly flows at the station of Saint Gervais.

\begin{tabular}{|c|c|c|c|c|c|c|c|}
\cline { 2 - 8 } \multicolumn{1}{c|}{} & \multicolumn{4}{c|}{ Débit interannuel } & \multicolumn{3}{c|}{$\%$ Variation / 1CO2 } \\
\cline { 2 - 8 } \multicolumn{1}{c|}{} & $\mathbf{1 C O 2}$ & Arpege & LMD1 & LMD2 & Arpege & LMD1 & LMD2 \\
\hline Janvier & 243 & 282 & 335 & 255 & 16.1 & 38.1 & 5.1 \\
\hline Fèvrier & 252 & 394 & 365 & 294 & 56.5 & 44.9 & 16.9 \\
\hline Mars & 276 & 434 & 346 & 318 & 57.0 & 25.3 & 1.9 .1 \\
\hline Avril & 338 & 509 & 371 & 363 & 50.8 & 9.9 & 7.6 \\
\hline Mai & 448 & 528 & 397 & 403 & 17.9 & -11.5 & -10.1 \\
\hline Juin & 505 & 448 & 347 & 387 & -11.2 & -31.2 & -23.2 \\
\hline Juillet & 407 & 288 & 244 & 290 & -29.3 & -40.1 & -28.7 \\
\hline Août & 272 & 158 & 151 & 179 & -42.1 & -44.7 & -34.4 \\
\hline Septembre & 220 & 111 & 111 & 125 & -49.6 & -49.5 & -4.3 .3 \\
\hline Octobre & 257 & 142 & 152 & 174 & -44.9 & -41.0 & -32.6 \\
\hline Novembre & 268 & 196 & 219 & 226 & -26.9 & -18.1 & -15.6 \\
\hline Décembre & 264 & 251 & 299 & 258 & -5.0 & 13.4 & -2.5 \\
\hline Année & 313 & 312 & 278 & 273 & -0.9 & -8.7 & -12.2 \\
\hline
\end{tabular}

persion dans les résultats (pour le scénario ARPEGE, augmentation de $+57 \%$, alors que pour les scénarios LMD1 et LMD2, augmentation res- pective de $+45 \%$ et $+17 \%$ ). Par contre, l'ensemble des scénarios est très cohérent sur la période estivale et automnale : le débit mensuel inte- 


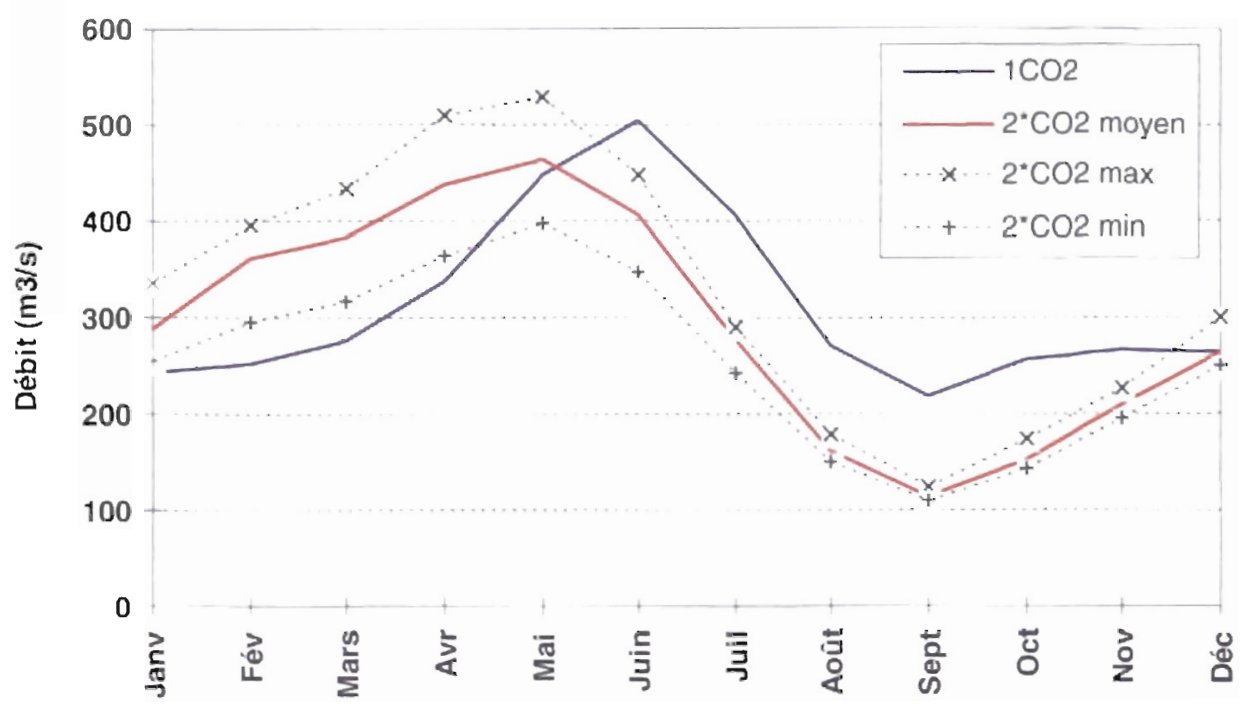

Fig. 9. - Évolution des débits mensuels interannuels à Saint Gervais le Port.

Fig. 9. - Evolution of mean monthly flow at Saint Gervais le Port.

Tableau IV. - Débits de crues estimés à St Gervais le Port (Isère) sur un échantillon de 25 ans par la méthode du renouvellement.

Table IV. - Flood discharge estimated by the renewal method at St Gervais le Port (Isere) from a 25-year sample.

\begin{tabular}{|c|c|c|c|c|}
\cline { 2 - 5 } \multicolumn{1}{c|}{} & \multicolumn{4}{c|}{ Débit de crue (m3/s) } \\
\hline Période de Retour & $1 \mathrm{mO2}$ & ARPEGE & LMD1 & LMD2 \\
\hline 10 ans & 951 & 1099 & 838 & 746 \\
50 ans & 1092 & 1261 & 974 & 811 \\
100 ans & 1152 & 1331 & 1032 & 839 \\
1000 ans & 1354 & 1562 & 1227 & 933 \\
\hline
\end{tabular}

rannuel du mois de septembre diminue de $-43 \%$ à $-50 \%$ selon le scénario utilisé.

Une première analyse sur les extrêmes permet de constater aussi des évolutions opposées (compte tenu du sens de variation) sur les crues. Sous $1{ }^{\star} \mathrm{CO}_{2}$, et avec l'échantillon disponible (25 ans), un calcul du débit de période de retour de 1000 ans avait donné $1354 \mathrm{~m}^{3} / \mathrm{s}$ avec la méthode du renouvellement (Miquel, 1984). Sous $2^{\star} \mathrm{CO}_{2}$, ce même débit calculé sur un nombre d'années équivalent devient $1562 \mathrm{~m}^{3} / \mathrm{s}$ sous ARPEGE, $1227 \mathrm{~m}^{3} / \mathrm{s}$ sous LMD1 et $933 \mathrm{~m}^{3} / \mathrm{s}$ sous LMD2 (tableau IV). Les scénarios LMD ont tendance à prédire une diminution de 
Tableau V. - Débits à Saint Gervais le Port (Isère) en fonction du \% temps de dépassement en moyenne sur les années.

Table V - Flow discharge exceeded during a mean percentage of the year at Saint Gervais le Port (Isère).

\begin{tabular}{|c|c|c|c|c|}
\hline $\begin{array}{c}\text { \% temps de } \\
\text { dépassement }\end{array}$ & 1 CO2 & ARPEGE & LMD1 & LMD2 \\
\hline 99.9 & 70 & 38 & 39 & 46 \\
99 & 97 & 50 & 51 & 59 \\
95 & 141 & 81 & 78 & 90 \\
90 & 168 & 100 & 103 & 117 \\
80 & 204 & 140 & 142 & 155 \\
70 & 233 & 178 & 182 & 191 \\
50 & 285 & 276 & 262 & 258 \\
30 & 349 & 377 & 350 & 327 \\
20 & 406 & 449 & 399 & 379 \\
10 & 500 & 583 & 469 & 446 \\
5 & 578 & 695 & 535 & 500 \\
1 & 760 & 870 & 638 & 638 \\
0.1 & 877 & 1013 & 763 & 717 \\
\hline
\end{tabular}

l'occurrence des crues, tandis que le scénario ARPEGE tend à annoncer leur renforcement (la centennale aurait la même valeur que la crue millénale sous $1^{*} \mathrm{CO}$,). Rappelons que les résultats de cette analyse sont assez dépendants de la méthode choisie pour désagréger le climat sous changement climatique.

Les débits minimums sont plus fortement perturbés, mais de manière cohérente entre les scénarios. Le débit dépassé $95 \%$ du temps (Q $95 \%$ ) sous $1{ }^{*} \mathrm{CO}_{2}$ est passé de $141 \mathrm{~m}^{3} / \mathrm{s}$ à 78,81 et $90 \mathrm{~m}^{3} / \mathrm{s}$ avec respectivement les scénarios LMD1, ARPEGE et LMD2 (tableau V). Si l'on prend le Q $95 \%$ sous $1{ }^{*} \mathrm{CO}_{2}$ (i.e. $141 \mathrm{~m}^{3} / \mathrm{s}$ ) qui correspond à un débit non dépassé 18 jours de l'année et que l'on regarde maintenant sous $2{ }^{\star} \mathrm{CO}_{2}$ pour ce même débit le nombre de jours non dépassés, on constate que celui-ci passe respectivement à 74,72 et
57 jours sur l'année avec les scénarios ARPEGE, LMD1 et LMD2. Ceci indique une beaucoup plus grande occurrence des faibles débits.

On assiste donc à une réelle modification des régimes hydrologiques sur le sous bassin versant de l'Isère. Le régime nival reste encore présent mais le maximum apparait un mois plus tôt. Le rôle du stock neigeux devient moindre par un cumul de neige sur l'hiver beaucoup plus faible et une durée d'enneigement réduite. Le soutien d'étiage jusqu'en automne, assuré par le manteau neigeux, perd de son efficacité et laisse apparaître des réductions de moitié des débits en septembre. En contrepartie, les débits hivernaux augmentent, en profitant de températures plus chaudes, voire comme dans le cas du scénario ARPEGE de précipitations plus abondantes. 
Tableau VI. - Temperatures moyennes de l'air du sous bassin de Viviers et variation en "C. Table VI. - Mean air temperature for the sub-catchment of Viviers and changes in "C.

\begin{tabular}{|c|c|c|c|c|c|c|c|}
\cline { 2 - 8 } \multicolumn{1}{c|}{} & \multicolumn{4}{c|}{ Température moyenne } & \multicolumn{3}{c|}{ Variation de température / 1CO2 } \\
\cline { 2 - 8 } \multicolumn{1}{c|}{} & $1 \mathrm{CO2}$ & ARPEGE & LMDI & LMD2 & ARPEGE & LMDI & LMD2 \\
\hline Janvier & 0.9 & 2.6 & 4.3 & 2.9 & 1.7 & 3.4 & $2.0)$ \\
\hline Février & 2.4 & 4.1 & 5.2 & 4.5 & 1.7 & 2.8 & 2.1 \\
\hline Mars & 4.8 & 6.8 & 6.1 & 6.6 & 2.0 & 1.2 & 1.8 \\
\hline Avril & 7.9 & 10.7 & 9.5 & 9.1 & 2.8 & 1.6 & 1.1 \\
\hline Mai & 11.9 & 13.4 & 12.8 & 13.1 & 1.4 & 0.9 & 1.2 \\
\hline Juin & 15.9 & 19.3 & 18.3 & 17.3 & 3.4 & 2.4 & 1.4 \\
\hline Juillet & 18.8 & 23.0 & 23.1 & 20.2 & 4.1 & 4.3 & 1.4 \\
\hline Août & 18.2 & 22.6 & 21.4 & 22.0 & 4.4 & 3.2 & 3.8 \\
\hline Septembre & 15.0 & 18.7 & 18.5 & 19.6 & 3.7 & 3.5 & 4.6 \\
\hline Octobre & 10.0 & 12.2 & 11.9 & 12.1 & 2.1 & 1.8 & 2.1 \\
\hline Novembre & 4.8 & 7.5 & 7.8 & 6.4 & 2.6 & 3.0 & 1.6 \\
\hline Décembre & 1.9 & 3.9 & 5.7 & 3.7 & 2.1 & 3.8 & 1.8 \\
\hline Année & 9.4 & 12.1 & 12.1 & 11.5 & 2.7 & 2.7 & 2.1 \\
\hline
\end{tabular}

\subsubsection{Evolutions à la station de Viviers : fin du domaine d'étude}

Les trois scénarios climatiques utilisés indiquent une augmentation moyenne de la température de 2,1 à $2,7^{\circ} \mathrm{C}$ sur le sous bassin versant de Viviers (tableau VI).

Cette augmentation de température se décline dans l'année par une élévation de température toujours supérieure à $1^{\text {" }} \mathrm{C}$ et pouvant atteindre $4,5{ }^{\circ} \mathrm{C}$ sur la période estivale.

Indépendamment des effets d'un changement climatique sur le bassin suisse, les lames d'eau annuelles écoulées seront peu modifiées (tableau VII). Le scénario ARPEGE n'entraîne quasiment pas de variation de la lame d'eau annuelle $(-1,4 \%)$ tandis que les scénarios LMD engendrent une diminution de l'ordre de $12 \%$.
La répartition sur l'année de la lame d'eau écoulée est, par contre, beaucoup plus marquée. Sur l'hiver, le scénario ARPEGE se démarque par des lames d'eau écoulées plus importantes (+69\% en février, $+26 \%$ en mars), tandis que les scénarios LMD r'annoncent que des variations de $+\grave{a}-10 \%$. Par contre, sur la période estivale, on retrouverait une certaine cohérence des résultats avec des diminutions importantes de -32 à $-39 \%$ en septembre pour les différents scénarios (fig. 10).

L'analyse des extrêmes, toujours par la méthode du renouvellement (Miquel, 1984), permet de constater des évolutions très différentes sur les crues. Sous $1^{*} \mathrm{CO}_{2}$, et avec l'échantilIon disponible, un calcul du débit de période de retour de 1000 ans avait donné $8124 \mathrm{~m}^{3} / \mathrm{s}$ (tableau VIII). Sous $2{ }^{*} \mathrm{CO}_{2}$, ce même débit calculé sur un 
Tableau VII. - Évolution des débits mensuels interannuels à la station de Viviers sans tenir compte d'une évolution sur la Suisse.

Table VII. - Change in mean monthly flows at Viviers simulated without change on the Swiss part of the catchment.

\begin{tabular}{|c|c|c|c|c|c|c|c|}
\cline { 2 - 8 } \multicolumn{1}{c|}{} & \multicolumn{4}{c|}{ Débit interannuel } & \multicolumn{3}{c|}{ \% Variation / 1CO2 } \\
\cline { 2 - 8 } \multicolumn{1}{c|}{} & 1c02 & Arpege & LMD1 & LMD2 & Arpege & LMD1 & LMD2 \\
\hline Janvier & 1648 & 1878 & 1950 & 1587 & 14.0 & 18.3 & -3.7 \\
\hline Février & 2059 & 3478 & 2192 & 2065 & 68.9 & 6.5 & 0.3 \\
\hline Mars & 1764 & 2223 & 1565 & 1652 & 26.0 & -11.3 & -6.3 \\
\hline Avril & 1856 & 2257 & 1660 & 1746 & 21.6 & -10.5 & -5.9 \\
\hline Mai & 1816 & 1964 & 1564 & 1663 & 8.1 & -13.9 & -8.4 \\
\hline Juin & 1690 & 1489 & 1276 & 1466 & -11.9 & -24.5 & -13.3 \\
\hline Juillet & 1330 & 1060 & 1021 & 1148 & -20.3 & -23.3 & -13.7 \\
\hline Aoôt & 982 & 729 & 737 & 779 & -25.8 & -25.0 & -20.7 \\
\hline Septembre & 940 & 575 & 597 & 641 & -38.8 & -36.5 & -31.9 \\
\hline Octobre & 1206 & 729 & 792 & 961 & -39.5 & -34.3 & -20.3 \\
\hline Novembre & 1299 & 1043 & 1147 & 1168 & -19.7 & -11.7 & -10.1 \\
\hline Décembre & 1642 & 1658 & 1843 & 1611 & 1.0 & 12.2 & -1.9 \\
\hline Année & 1519 & 1590 & 1362 & 1374 & -1.4 & -12.8 & -11.3 \\
\hline
\end{tabular}

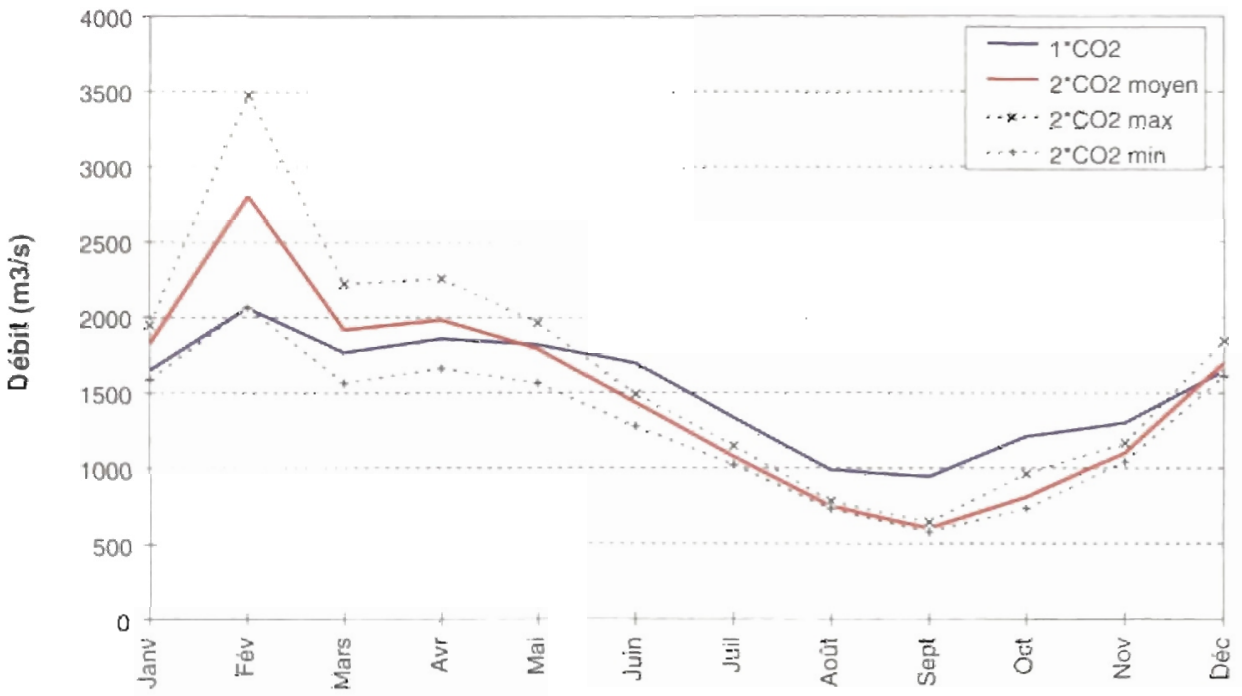

Fig. 10. - Evolution des débits mensuels interannuels à Viviers, sans tenir compte d'une évolution sur la Suisse

Fig. 10. - Evolution of mean monthly flow at: Viviers. 
Tableau VIII.- Débits de crues estimés à Viviers sur un échantillon de 25 ans par la méthode du renouvellement.

Table VIII. - Flood discharge estimated by the renewal method at Viviers for a 25-year sample.

\begin{tabular}{|c|c|c|c|c|}
\cline { 2 - 5 } \multicolumn{1}{c|}{} & \multicolumn{4}{c|}{ Débit de crue (m3/s) } \\
\hline Période de Retour & 1 CO2 & ARPEGE & LMD1 & LMD2 \\
\hline 10 ans & 5469 & 9411 & 5811 & 5613 \\
50 ans & 6397 & 11923 & 6975 & 6769 \\
100 ans & 6796 & 13005 & 7477 & 7267 \\
1000 ans & 8124 & 16599 & 9142 & 8922 \\
\hline
\end{tabular}

nombre d'années équivalent deviendrait $16600 \mathrm{~m}^{3} / \mathrm{s}$ sous ARPEGE, correspondant à un doublement du débit millénal, et $9142 \mathrm{~m}^{3} / \mathrm{s}$ et $8922 \mathrm{~m}^{3} / \mathrm{s}$ respectivement sous LMD1 et LMD2. Les scénarios LMD ont donc tendance à prédire un renforcement significatif d'environ $10 \%$ des débits de crues $\left(+800\right.$ à $1000 \mathrm{~m}^{3} / \mathrm{s}$, à un niveau de crue millénale), tandis que le scénario ARPEGE, tend à annoncer un très fort développement des épisodes de crues (la décennale sous $2^{\star} \mathrm{CO}_{2}$, serait supérieure à la valeur de la millénale sous $1^{\star} \mathrm{CO}_{2}$ ), via un fort renforcement des précipitations hivernales.

Ces résultats sont toujours très liés à la méthode retenue pour la désagrégation de l'information climatique, à savoir la méthode des perturbations. Ils soulèvent toutefois deux interrogations : le modèle hydrologique est-il encore valable dans cette plage de débits jamais observés dans le passé? De telles précipitations ont-elles encore un sens physique?

Les débits minimums sont eux aussi modifiés et de manière cohérente entre les scénarios (tableau IX). Le

Tableau IX. - Débits à Viviers en fonction du \% temps de dépassement annuel.

Table IX - Flow discharge exceeded during a mean percentage of the year at Viviers.

\begin{tabular}{|c|c|c|c|c|}
\hline $\begin{array}{c}\text { \% temps de } \\
\text { dépassement }\end{array}$ & 1 CO2 & ARPEGE & LMD1 & LMD2 \\
\hline 99.9 & 346 & 255 & 245 & 261 \\
99 & 404 & 294 & 295 & 309 \\
95 & 566 & 415 & 420 & 438 \\
90 & 707 & 518 & 522 & 542 \\
80 & 876 & 677 & 681 & 702 \\
70 & 1019 & 840 & 814 & 832 \\
50 & 1359 & 1248 & 1150 & 1155 \\
30 & 1795 & 1792 & 1548 & 1543 \\
20 & 2051 & 2226 & 1876 & 1851 \\
10 & 2499 & 2981 & 2422 & 2348 \\
5 & 3042 & 3890 & 3167 & 3043 \\
1 & 4267 & 6512 & 4465 & 4320 \\
0.1 & 5810 & 8998 & 5885 & 5611 \\
\hline
\end{tabular}


débit dépassé $95 \%$ du temps ( $Q$ $95 \%$ ) sous $1{ }^{*} \mathrm{CO}_{2}$ est passé de $566 \mathrm{~m}^{3} / \mathrm{s}$ à 415,420 et $438 \mathrm{~m}^{3} / \mathrm{s}$ avec respectivement les scénarios ARPEGE, LMD1 et LMD2. Si l'on prend le Q $95 \%$ sous $1{ }^{*} \mathrm{CO}_{2}$ (i.e. $566 \mathrm{~m}^{3} / \mathrm{s}$ ) qui correspond à un débit non dépassé 18 jours de l'année et que l'on regarde maintenant sous $2^{*} \mathrm{CO}_{2}$ pour ce même débit le nombre de jours non dépassé, on constate que celui-ci passe à 47,45 et 41 jours avec respectivement les scénarios ARPEGE, LMD1 et LMD2. Ceci indique une plus grande occurrence des faibles débits.

On assiste donc à une réelle modification des régimes hydrologiques à la station de Viviers. L'Isère perdant une partie de son fonctionnement actuel en régime nival (crue l'été) au profit d'un fonctionnement de type pluvial (crue l'hiver), on constate une plus grande corrélation entre les crues issues du bassin de la Saône et celles issues du bassin de l'Isère, renforçant d'autant les crues en aval du bassin.

De même, le bassin du Rhône situé en Suisse n'ayant pas été modélisé dans cette étude faute de données disponibles, il a été tenté de tenir compte globalement d'un changement climatique analogue sur la Suisse. La limite à cette hypothèse est liée à la capacité du lac Léman à influer les volumes mensuels écoulés, en fonction de ses règles de gestion.

En tenant compte d'une modification similaire des débits entre bassin suisse et bassin de l'Isère, en raison de leur proximité géographique et leurs similitudes climatiques, on constate que la lame d'eau annuelle serait toujours très peu modifiée par le scénario ARPEGE. Par contre, les scénarios LMD réduiraient la lame d'eau annuelle de plus de $15 \%$ (tableau $X$ ), ce qui constituerait une diminution conséquente.

La modification de la répartition des volumes écoulés dans l'année se trouverait renforcée. Cela se traduirait notamment par des étiages encore plus sévères avec des variations allant de -45 à $-55 \%$ sur le débit mensuel interannuel du mois de septembre.

\subsection{Limites de l'analyse hydrologique du changement climatique}

Les résultats précédents restent soumis à deux hypothèses majeures, qu'il conviendra de confirmer :

- Hypothèse 1 : les précipitations se renforcent en intensité et non en occurrence, ce qui a conduit à utiliser la méthode des perturbations,

- Hypothèse 2 : les modèles hydrologiques des sept sous bassins versants sont suffisamment robustes pour sortir de leur plage de calage et garder leur capacité à représenter correctement l'hydrologie continentale,

et à d'autres hypothèses implicites :

- le changement de climat n'influe pas sur une évolution de la végétation, 
Tableau X. - Évolution des débits mensuels interannuels à la station de Viviers en tenant compte d'un changement similaire sur le bassin du Rhône en Suisse.

Table X. - Change in mean monthly flows at Viviers with similar change on the Swiss part of the catchment.

\begin{tabular}{|c|c|c|c|c|c|c|c|}
\hline & \multicolumn{4}{|c|}{ Débit interannuel } & \multicolumn{3}{|c|}{$\%$ Variation / 1 CO2 } \\
\hline & $1 \mathrm{CO} 2$ & Arpege & LMD1 & LMD2 & Arpege & LMD1 & LMD2 \\
\hline Janvier & 1648 & 1919 & 2049 & 1600 & 16.4 & 24.3 & -22 \\
\hline Février & 2059 & 3653 & 2337 & 2118 & 774 & 13.5 & 29 \\
\hline Mars & 1764 & 2390 & 1640 & 1697 & 35.5 & .70 & -3.8 \\
\hline Avril & 1856 & 2425 & 1696 & 1773 & 30.6 & -8.6 & -4.5 \\
\hline Mai & 1816 & 2033 & 1526 & 1628 & 112 & -16.0 & -10.4 \\
\hline Juin & 1690 & 1440 & 1125 & 1353 & -14.8 & -33.4 & -104 \\
\hline Juillet & 1330 & 913 & 815 & 998 & -31.4 & -38.7 & -250 \\
\hline Aoūt & 982 & 560 & 554 & 641 & -43.0 & -43.6 & -34.8 \\
\hline Septembre & 940 & 417 & 438 & 505 & -55.6 & .53 .4 & -46.3 \\
\hline Octobre & 1206 & 608 & 681 & 871 & -49.5 & -43.5 & -27.8 \\
\hline Novembre & 1299 & 979 & 1103 & 1129 & -24.6 & -15.1 & $-13,1$ \\
\hline Décembre & 1642 & 1642 & 1872 & 1604 & (1).1) & 14.1) & -2.4 \\
\hline Année & 1519 & 1582 & 1320 & 1326 & -3.9 & -17.3 & -15.7 \\
\hline
\end{tabular}

- le changement de climat n'influe pas sur les prélèvements d'eau (par irrigation entre autres),

- le changement de climat n'influe pas sur la gestion des ouvrages hydrauliques (barrages-réservoirs, Lac Léman).

Une évolution de la végétation pourrait modifier la répartition des précipitations entre le domaine souterrain, les écoulements de surface et ce qui repart dans l'atmosphère. Ceci pourrait jouer sur la capacité des nappes à jouer leur rôle de soutien d'étiage.

Une augmentation de la température et des sécheresses peut tendre à augmenter le besoin en eau pour l'agriculture. Ceci pourrait renforcer l'affaiblissement des débits estivaux.

La gestion de l'eau, au travers des différents stockages réalisés au sein du bassin versant, pourrait en partie modifier les effets du changement climatique en jouant sur un déplacement dans l'année des volumes d'eau disponible pour l'écoulement.

\section{CONCLUSION}

Un modèle hydrologique a été déployé sur l'ensemble du bassin du Rhône permettant de mettre en relation la météorologie de l'ensemble du bassin versant français avec la ressource en eau.

L'utilisation conjointe de ce modèle hydrologique (découpé en sept sous modèles) et de trois scénarios de changement climatique, établis à partir de 2 modèles différents, a permis de faire une première évaluation des tendances à attendre sur la dis- 
ponibilité de la ressource en eau d'ici à 50 ans en différents points du bassin du Rhône.

Les trois scénarios utilisés permettent d'obtenir les premiers résultats suivants :

- Les trois scénarios de changement de climat utilisés pour cette étude indiquent tous une augmentation des températures moyennes sur l'année entre +2 et $+3^{\circ} \mathrm{C}$. Cette élévation se déclinerait sur l'année par une élévation en moyenne mensuelle interannuelle toujours supérieure à $+1^{\circ} \mathrm{C}$ et pouvant atteindre $+5^{\circ} \mathrm{C}$ en fin d'été, début d'automne.

- Le scénario ARPEGE ne modifierait quasiment pas la lame d'eau annuelle écoulée sur l'axe Rhône. Par contre, les scénarios LMD réduiraient significativement la lame d'eau annuelle écoulée sur l'axe Rhône $(-10$ à $-15 \%$ selon la station considérée).

- Les trois scénarios sont cohérents sur une aggravation des étiages avec une réduction des débits moyens mensuels interannuels d'étiage de l'ordre de 40 à $50 \%$ sur le Rhône en aval de Lyon, avec une forte extension des périodes d'étiages (par 2 à 4).

- Enfin, le scénario ARPEGE tendrait à annoncer systématiquement un fort renforcement des débits et des crues en hiver et notamment au mois de février $(+\sim 70 \%$ sur le débit mensuel interannuel de février). La crue décennale sous changement de climat ARPEGE correspondrait environ à la crue millénale sous climat actuel à la station de Viviers (cette analyse reste limitée à l'hypothèse d'un renforcement en intensité des précipitations plutôt que du nombre d'épisodes pluvieux). Par contre, les augmentations de débits sur la période hivernale pour le scénario LMD1 seraient de l'ordre de $20 \%$ pour le débit mensuel interannuel de janvier en aval de Lyon. Le scénario LMD2 n'entraînerait pour sa part quasiment aucune modification des débits hivernaux (inférieur à $5 \%$ ).

L'analyse précédente apporte des éléments majeurs de modifications des régimes hydrologiques sur l'axe Rhône: des étiages plus sévères (en débit et durée), un affaiblissement des régimes nivaux du massif alpin et des crues peut-être plus sévères sur la période hivernale. Cette analyse reste toutefois très liée aux hypothèses formulées et notamment celle retenue pour la désagrégation de l'information climatique (méthode des perturbations).

Si les cohérences entre les trois scénarios donnent aujourd'hui l'espoir d'avoir obtenu une évaluation fiable des évolutions de la contrainte hydrique, les divergences posent quant à elles la question de la fiabilité des hypothèses formulées, et des différents scénarios. L'approche retenue par ce travail n'a pas été de faire prévaloir un scénario plus qu'un autre mais bien de les considérer comme un ensemble de possibles.

II a donc été prévu que cette première analyse fasse l'objet de différents compléments dans les années 
à venir portant notamment sur les points suivants :

- Analyse de l'échauffement des eaux sous changement climatique,

- Extension de l'analyse à un autre grand bassin versant français : la Loire,

- Approfondissement des incertitudes par l'extension du nombre de scénarios pour la simulation sous changement climatique,

- Analyse de la sensibilité du comportement du modèle hydrologique sous changement climatique par rapport au calage retenu sur le climat actuel.

\section{RÉFÉRENCES BIBLIOGRAPHIQUES}

Charbonneau R., Fortin J.P. \& Morin G., 1977. The CEQUEAU Model : description and examples of its use in problemes related to water ressource management, Bull. des sc. Hydrologiques, 22 : 93-202.

IPCC, 2001. Climate change 2001. The scientifc basis - Summary for policy makers. Report of working group I of IPCC : $98 \mathrm{p}$.

Joussaume S., 1993. Climat d'hier à demain. Coll. Science au Présent : $144 \mathrm{p}$.

Martin E. Modélisation de la climatologie nivale des Alpes françaises: Application des techniques de régionalisation à l'étude de l'impact d'un changement climatique sur l'enneigement. Thèse de l'université Paul Sabatier, France, soutenue le 3 mai 1995 : 244 p.

Miquel J., 1984. Guide pratique d'estimation des probabilités de crues. Ed. Eyrolles : $165 \mathrm{p}$.

Nash J.E. \& Sutcliffe J.V., 1970. River flow forecasting through conceptual models, part 1 : a discussion of principles, Journal of Hydrology, 10(3), 282-290.

Parey S., 1996. Doublement de la concentration en $\mathrm{CO} 2$ atmosphérique et cycle hydrologique en Europe et en France avec les modèles du LMD et ARPEGE-CLIMAT. Rapport HE-33/96/ 010, Electricité de France : $87 \mathrm{p}$.

Reynard N.S., Prudhomme C. \& Crooks S., 2001. The flood characteristics of large UK rivers: potential effects of changing climate and land use, Climatic Change, 43, 343-359. 